There are several possible starting points for protein identification. But the most well-travelled route into proteomics starts with a sample in a 2D gel being fed into an electrophoresis machine. This is followed by either automatic or manual picking and excision of the protein spots of interest, which are then fed into a mass spectrometer (see 'Mix and match', previous page).

Celia Caulcott, who heads an effort by the UK's Biotechnology and Biological Sciences Research Council to develop new proteomics technologies, says that, despite a lot of R\&D, traditional techniques for protein identification still stand. "The gels still seem to be the pre-eminent way people want to do things," she says. Beguiling techniques such as protein arrays, which could supplant gels if successful, have yet to prove they can be viable both scientifically and commercially, she says.

Joakim Rodin, director for proteomics R\&D at Amersham Biosciences, a biotechequipment company based in Uppsala, Sweden, agrees that the gel system, although not the easiest thing to work with, has yet to be supplanted. "It's still a lot of work running the gels," he says. But improvements in capacity, such as the company's Ettan Dalt II system, allows researchers to run up to 12 gels in parallel with more reproducibility and sensitivity.

And the gels themselves have improved,

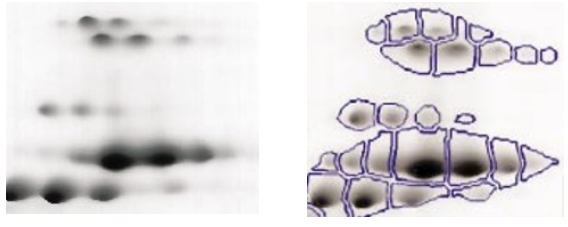

Identifying spots on gels can be time consuming.

he says. They are getting bigger, so more sample can be loaded, which improves the detection of low-abundance proteins.

'Zoom' gels have also been developed with ever-narrowing $\mathrm{pH}$ ranges, which give better resolution as well as higher sensitivity.

Fluorescent labelling is also getting better, he says. Differential-expression analysis using difference gel electrophoresis, developed at Carnegie Mellon University, allows up to three samples to be run simultaneously on a single gel using cyanine-dye chemistry. This should let researchers detect protein differences between normal and cancerous tissues on the same gel. The method also allows multiplexing of gels, which significantly increases throughput, reproducibility and accuracy. Multiple gels provide comparative analysis and accurate measurement of differential protein expression. Although the handling and analysis of 2D gels have improved dramatically, Rodin notes that complementary techniques, such as X-ray crystallography, are needed to resolve the whole proteome.

Fortunately, the next stage of the proteomics pipeline, handling the intermediate steps between electrophoresis and mass spectrometry, is becoming easier. Picking the protein spots off the gels, then digesting them into peptide fragments used to be two separate, manual tasks. Now they are becoming automated and are being integrated into the workflow (see 'Multiple choice', below). But improving and combining individual components can be challenging, says Steve Martin, director of Applied Biosystems' Proteomics Research Center in Framingham,

Massachusetts. For example, increasing the capacity of one instrument without accounting for the additional need for throughput in others can actually result in bottlenecks, he says.

Three commercial — and by today's standards, integrated — systems are made by Amersham Biosciences, Genomic Solutions in Ann Arbor, Michigan, and Bio-Rad in Hercules, California. Their basic components are similar - they all use robotic sample-preparation, 2D-gel electrophoresis, excision of spots, labelling, and ionization and analysis of the peptide fragments by mass spectrometry. In these systems, data generated from all the instruments are presented in a userfriendly graphical interface.

\title{
AUTOMATION
}

\section{Multiple choice}

U ntil recently, characterizing proteins was done slowly. But with the many candidates in the newly sequenced genomes crying out for attention, and the lure of complex protein assemblies beckoning, labs are gearing up to look at many proteins simultaneously.

The key to making such a system work lies in replacing error-prone humans with spot-picking robots, guided by cameras and sophisticated image-analysis software. The Australian company ARRM has a system that excises spots from gels or polyvinylidene fluoride membranes and places them in a 96-well plate for

subsequent proteolysis.

Genetix, of New Milton, UK, uses a line of sample-preparation, gel-spotting and spot-excision units. Soon these will be joined by a machine to prepare MALDI samples automatically, thus helping to integrate raw samples and mass fingerprints. Genetix is also getting into chip arrays and yeast two-hybrid systems, two automated ways of looking at protein interactions. Other major players in lab automation are Amersham Biosciences in Uppsala,

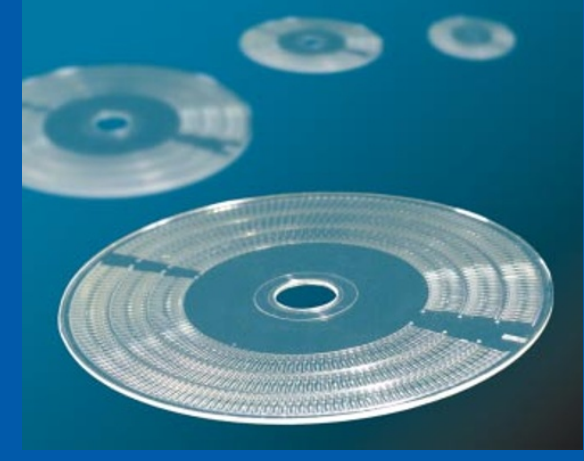

Lab-on-a-CD systems from Gyros.
Sweden, Bio-Rad in Hercules, California, and Genomic Solutions in Ann Arbor, Michigan.

Large Scale Biology in Germantown, Maryland, and Oxford Glycoscience in Cambridge, UK, aim to automate the entire protein-discovery process in humidity-controlled, robot-populated buildings. Here massive amounts of samples would travel through the pipeline from gel to mass spectrometer and data.

But harking back to the idea that small is beautiful, another school of thought sees a nano future for the science in 'labon-a-chip' technologies such as those of Caliper, of Fremont, California, and Gyros in Uppsala. Gyros has updated an idea from the 1970s by engraving microscale channels and mixing chambers on a compact disc. Centrifugal force and controlled surface chemistry are used to regulate the flow of liquid through the CD. Despite the small size of the system, the price tag will probably ensure that it will mainly be used by big pharmaceutical companies or 'protein factories' rather than small independent labs. 